\title{
Novel Y Chromosome Retrocopies in Canids Revealed through a Genome-Wide Association Study for Sex
}

\author{
Kate L. Tsai ${ }^{1}$, Jacquelyn M. Evans ${ }^{1,2}$, Rooksana E. Noorai ${ }^{3}{ }^{\circledR}$, Alison N. Starr-Moss ${ }^{1}$ and \\ Leigh Anne Clark ${ }^{1, *}$ \\ 1 Department of Genetics and Biochemistry, Clemson University, Clemson, SC 29634, USA; \\ ktsai@clemson.edu (K.L.T.); jacquelyn.evans@nih.gov (J.M.E.); astarr@clemson.edu (A.N.S.-M.) \\ 2 Cancer Genetics and Comparative Genomics Branch, National Human Genome Research Institute, National \\ Institutes of Health, Bethesda, MD 20892-2152, USA \\ 3 Clemson University Genomics and Bioinformatics Facility, Clemson University, Clemson, SC 29634, USA; \\ rooksan@clemson.edu \\ * Correspondence: lclark4@clemson.edu
}

Received: 25 March 2019; Accepted: 18 April 2019; Published: 25 April 2019

check for updates

\begin{abstract}
The lack of an annotated reference sequence for the canine $\mathrm{Y}$ chromosome has limited evolutionary studies, as well as our understanding of the role of Y-linked sequences in phenotypes with a sex bias. In genome-wide association studies (GWASs), we observed spurious associations with autosomal SNPs when sex was unbalanced in case-control cohorts and hypothesized that a subset of SNPs mapped to autosomes are in fact sex-linked. Using the Illumina 230K CanineHD array in a GWAS for sex, we identified SNPs that amplify in both sexes but possess significant allele frequency differences between males and females. We found 48 SNPs mapping to 14 regions of eight autosomes and the $\mathrm{X}$ chromosome that are $\mathrm{Y}$-linked, appearing heterozygous in males and monomorphic in females. Within these 14 regions are eight genes: three autosomal and five $X$-linked. We investigated the autosomal genes (MITF, PPP2CB, and WNK1) and determined that the SNPs are diverged nucleotides in retrocopies that have transposed to the $\mathrm{Y}$ chromosome. MITFY and WNK1Y are expressed and appeared recently in the Canidae lineage, whereas PPP2CBY represents a much older insertion with no evidence of expression in the dog. This work reveals novel canid $Y$ chromosome sequences and provides evidence for gene transposition to the $Y$ from autosomes and the $X$.
\end{abstract}

Keywords: dog; canine; MITF; PPP2CB; WNK1; ETV5; retrogene

\section{Introduction}

The $\mathrm{Y}$ chromosome is historically described as a degenerate $\mathrm{X}$ chromosome that has lost about $95 \%$ of its ancestral genes and is poorly conserved among mammals [1,2]. Evolutionary compensation for gene loss on the $\mathrm{Y}$ is proposed to occur through the transposition and retrotransposition of genes from the $\mathrm{Y}$ chromosome to other chromosomes [3,4]. In the opposite direction, the $\mathrm{Y}$ chromosome has acquired new genetic material from the $X$ chromosome or autosomes through the same mechanisms [5-9]. Novel Y-linked genes usually have testis-specific expression and function in spermatogenesis, likely conferring a reproductive advantage in males $[1,8,10,11]$.

Sequence for the $\mathrm{Y}$ chromosome is not readily available for many organisms that have an otherwise complete reference genome. The 7.6X canine reference genome was generated from a female boxer, with $>98 \%$ of assembled sequence ordered and oriented on 39 nuclear chromosomes and 3228 unplaced scaffolds [12,13]. Having been excluded from the reference genome, ascertainment of canine $\mathrm{Y}$ chromosome sequences has been the focus of subsequent studies. Approximately $24 \mathrm{~kb}$ of $\mathrm{Y}$ 
chromosome sequences were determined through the identification of shotgun reads that 1) possessed sequence similarity to the human $\mathrm{Y}$ chromosome and 2) were present in a 1.5X sequence from a male poodle, but not the female reference genome [14]. A 2013 study of the canine Y chromosome utilized expressed sequences and a Doberman BAC library to capture novel $Y$ sequences and annotate male-specific genes, resulting in the most complete $\mathrm{Y}$ chromosome reference sequence available to date [15].

Today, commercially-available canine SNP arrays include Y chromosome markers; however, the arrays were initially developed using highly polymorphic SNPs identified from the reference genome and lower coverage sequences ( 1-1.5X) from 10 dog breeds, 4 wolves, and a coyote [12]. Genomes from male dogs sequenced for SNP discovery included reads from the Y chromosome, which presumably remained unmapped. While performing genome-wide association studies (GWASs) for complex traits using SNP data generated from the Illumina 230K CanineHD array, we observed spurious associations with autosomal SNPs when sex was not balanced between cases and controls. This led us to hypothesize that some SNPs placed on autosomes are sex-linked. Here, we test our hypothesis by performing a GWAS for sex.

\section{Materials and Methods}

Genomic DNAs and tissues used for this work were obtained with protocols approved by the Clemson University Institutional Review Board (IBC2008-17). Genotyping was performed for 72 German shepherd dogs using the Illumina CanineHD 230K BeadChip. SNPs having call rates $<95 \%$ and minor allele frequencies $<5 \%$ were removed, leaving 123,247 SNPs. We used 25 females and 25 males having call rates $>97 \%$ in a case-control analysis. Fisher's exact $P$-values were calculated under a dominant model. Filtering and statistical analyses were performed with SNP \& Variation Suite v8 (SVS, Golden Helix, Bozeman, MT).

Whole genome sequences from domestic dogs were available in our lab; all other Canidae genomes (Table S1) were downloaded from NCBI's SRA database using fasterq-dump from sratoolkit v2.9.2 (https://ncbi.github.io/sra-tools/). The quality of reads was assessed by FastQC v0.11.6 (https: //www.bioinformatics.babraham.ac.uk/projects/fastqc/) before and after trimmomatic v0.36 [16] was run to remove low quality bases and adapter sequences. Gsnap v2018-05-30 [17] was used to align fastq files to the canFam 3.1 genome using default parameters. Samtools v1.6 [18] was used to convert, sort, and index the alignment files for review in Integrative Genomics Viewer (IGV) v2.4.14 [19]. Using IGV, we searched for evidence of the $\mathrm{Y}$ chromosome duplications and, in the autosomal expressed regions, we evaluated at least one male and one female from each species. When we observed characteristics of a duplication in the male, but not the female, and identified more than 2 shared polymorphisms with the Canis familiaris $\mathrm{Y}$ chromosome copy, we posited that an ancestral version of the retrocopy was present. Retrocopies and sequences from unmapped mate pairs were analyzed for similarities with the canine Y assembly (GenBank: KP081776.1) using NCBI BLAST.

To check for expression and splicing activity, we utilized archival heart, brain, and liver tissues obtained from intact male dogs. Total RNA was isolated using the ToTALLY RNA Total Cellular RNA Isolation kit (Ambion, Foster City, CA). We obtained dog testis RNA from Zyagen (San Diego, CA). To remove and digest genomic DNAs, the RNAs were treated using the TURBO DNA-free kit (Invitrogen, Carlsbad, CA), following the manufacturer's instructions for rigorous treatment. cDNAs were synthesized with an oligo dT primer using the RevertAid First Strand cDNA Synthesis kit (Thermo Scientific, Pittsburgh, PA). For each tissue, a genomic control was run, to which no reverse transcriptase was added.

Primers to amplify the MITF, PPP2CB, and WNK1/ETV5 Y chromosome retrocopies were designed in diverged sequences, when possible, and with forward and reverse primers in different exons. Primer sequences were: MITFY: F 5'-CAGGTGCCGATGGAAGT, R 5'-TGAAGGAGGTCTTGGTTGCT; PPP2CBY: F 5'-CTGAACGAGAACCAAGTGT, R 5'-CACCAAATAGGATGTAATGACAA; WNK1Y: F 5'-CTTTCAAGGAGAAGAAGGCG, R 5'-CAACCTGTCCCACTCTACTGAT. A fourth primer set uses 
an ETV5Y F (5'-CTTTCTGTCAATCACATGCCA) with WNK1Y-R. Primer sets to amplify a total of 1,879 bp of chrUn_AAEX03024224 were: ChUn1: F 5'-GTGGTGCTGACCCTGTGC; R 5'-TTCCCTGG TATGAGTGTCTGTG; ChUn2: F 5'-TGGGTGATTTGTAGATGGTAGG; R 5' -GGGTTGAGGGAATGG TGAA; ChUn3: F 5'-GAGGGTGGAGGGTCTGCT; R 5'-AACACAAGGGTAAATCAGGTCAC. Genomic DNAs from three female and three male German shepherd dogs were used to test for male-specific amplification. All PCRs were performed using Phire Green Hot Start II DNA Polymerase mix (Thermo Scientific) following manufacturer guidelines for a $20 \mu \mathrm{L}$ reaction. Products were cleaned with ExoSAP-IT PCR Product Cleanup (Applied Biosystems, Foster City, CA), and sequence was confirmed using Sanger sequencing.

\section{Results}

A GWAS for sex identified 50 genome-wide significant SNPs with identical $P$-values of $1.58 \times 10^{-14}$, mapping to eight autosomes and both sex chromosomes (Table 1). A Manhattan plot of the results shows no evidence of linkage disequilibrium decay at the identified loci, usually illustrated by proximal SNPs with increasingly larger $P$-values supporting the lead SNP, except in the pseudoautosomal region of the $\mathrm{X}$ chromosome (Figure 1). Inspection of genotypes for the 50 SNPs revealed all males to be heterozygous and all females to be monoallelic, except for chrX:60395963 in PGK1 for which some males were homozygous. This SNP was excluded from further study. Of the remaining 47 non-Y chromosome SNPs, 19 are located within seven genes, 22 are intergenic, five are telomeric, and one is centromeric.

Table 1. Genome-wide significant markers identified in a GWAS for sex $\left(P\right.$-value $\left.=1.58 \times 10^{-14}\right)$.

\begin{tabular}{|c|c|c|c|c|c|c|c|}
\hline Chr & Position & Gene & Region & Chr & Position & Gene & Region \\
\hline \multirow[t]{2}{*}{6} & 72952463 & & & 20 & 21870155 & MITF & exonic \\
\hline & 72964379 & & & & 21870230 & MITF & exonic \\
\hline \multirow[t]{2}{*}{15} & 111194 & & & & 21870310 & MITF & exonic \\
\hline & 29973349 & & & & 21870623 & MITF & $3^{\prime}$ UTR \\
\hline \multirow[t]{2}{*}{16} & 33566554 & РPР2CB & $3^{\prime}$ UTR & & 21871904 & MITF & $3^{\prime}$ UTR \\
\hline & 33566935 & РPР2CB & $3^{\prime}$ UTR & & 21872335 & MITF & $3^{\prime}$ UTR \\
\hline 17 & 64209287 & & & & 21872815 & MITF & $3^{\prime}$ UTR \\
\hline \multirow[t]{18}{*}{19} & 20034966 & & & & 21873532 & MITF & $3^{\prime}$ UTR \\
\hline & 20040153 & & & 27 & 42911747 & WNK1 & exonic \\
\hline & 20052361 & & & & 42911917 & WNK1 & exonic \\
\hline & 20062503 & & & 32 & 38734101 & & \\
\hline & 20072295 & & & & 38767343 & & \\
\hline & 20100030 & & & & 38788489 & & \\
\hline & 20114792 & & & & 38789367 & & \\
\hline & 20130159 & & & $X$ & 6604781 & SHROOM2 & exonic \\
\hline & 20152245 & & & & 6621021 & SHROOM2 & intronic \\
\hline & 20172164 & & & & 6628533 & SHROOM2 & intronic \\
\hline & 20245553 & & & & 6634742 & SHROOM2 & intronic \\
\hline & 20256174 & & & & 10131021 & TRAPPC2 & exonic \\
\hline & 20286580 & & & & 10175834 & OFD1 & exonic \\
\hline & 20292945 & & & & 35604689 & USP9XN & exonic \\
\hline & 20303661 & & & & 57139861 & & \\
\hline & 20309777 & & & & 60395963 & PGK1 & \\
\hline & 20310190 & & & $\mathrm{Y}$ & 26641 & & \\
\hline & 20314276 & & & & 316950 & & \\
\hline
\end{tabular}




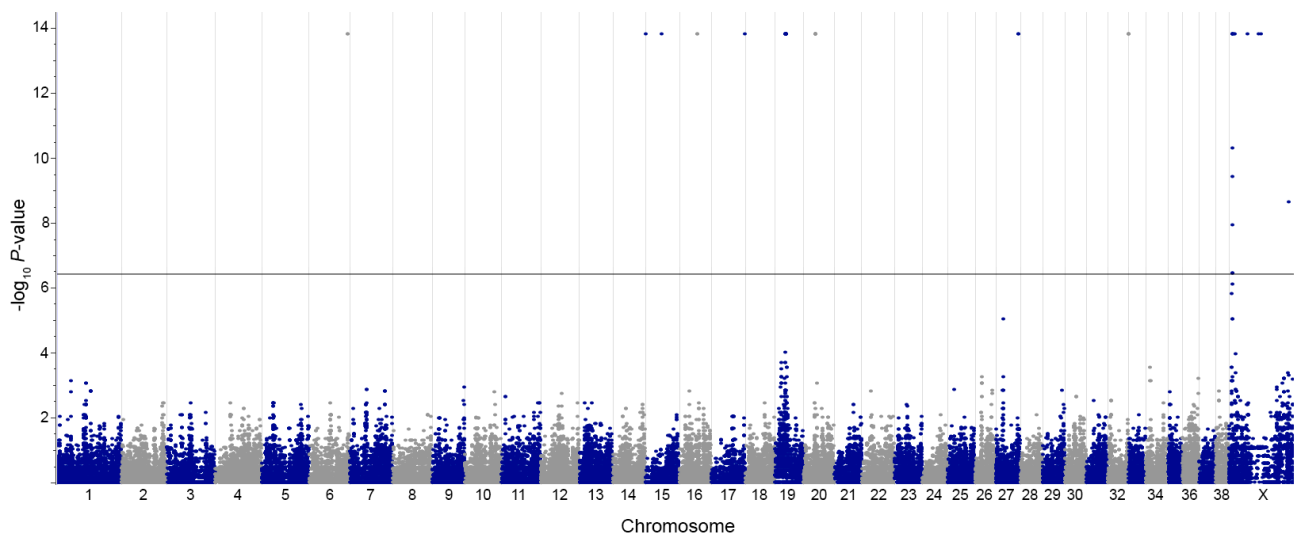

Figure 1. Manhattan plot illustrating results for a genome-wide association study for sex. The $-\log _{10}$ $P$-value (y-axis) is plotted against chromosome position (x-axis). The black horizontal line marks the threshold for Bonferroni significance.

We further investigated the SNPs mapping to exonic or untranslated regions of the autosomal genes MITF, $P P P 2 C B$, and WNK1. Using next generation sequencing reads aligned to the reference genome, we visually inspected each gene. Within the exons of male dogs only, we observed: distinct increases in sequence coverage, heterozygosity, unmapped mate pairs, and reads with low mapping scores, an indication that they map to multiple locations (Figure 2). Taken together, these findings led us to posit that the SNPs are present on diverged retrocopies located on the $Y$ chromosome. To test our hypothesis, we used next generation sequencing data to design PCR primers that spanned several exons, such that only a retrocopy would amplify from genomic DNA. PCR with gDNA showed male-specific amplification for each of the three genes (Figure 3), confirming their location on the $Y$ chromosome. We termed the three retrocopies: MITFY, PPP2CBY, and WNK1Y. The retrocopies and flanking sequences (determined using unmapped mate pairs) showed no significant similarities to the canine $\mathrm{Y}$ assembly.

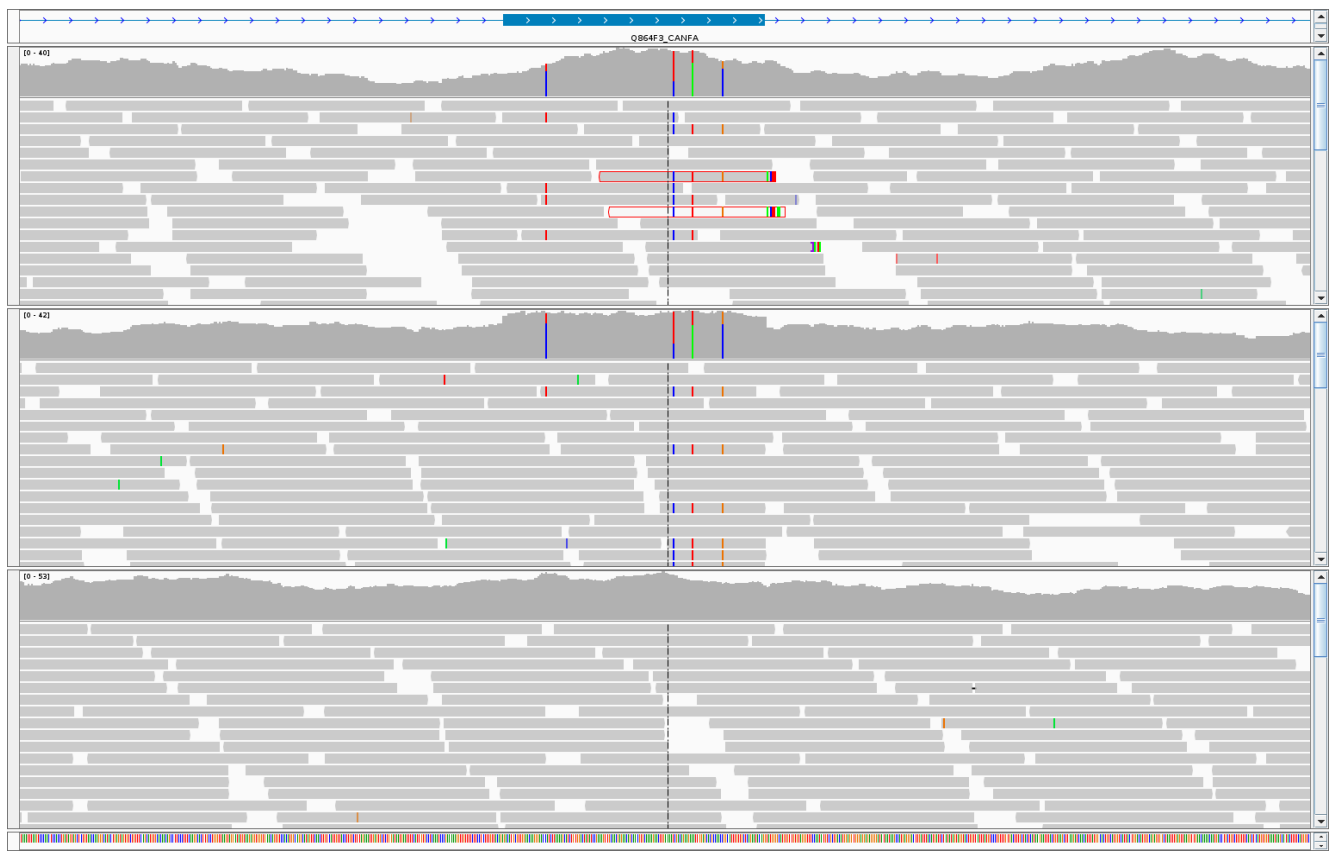

Figure 2. Integrative Genomics Viewer screenshot of a MITF exon illustrating increased read coverage, unmapped mate pairs (red outline), low mapping score (white read), and increased heterozygosity in male dogs (top and middle) as compared to a female (bottom). 


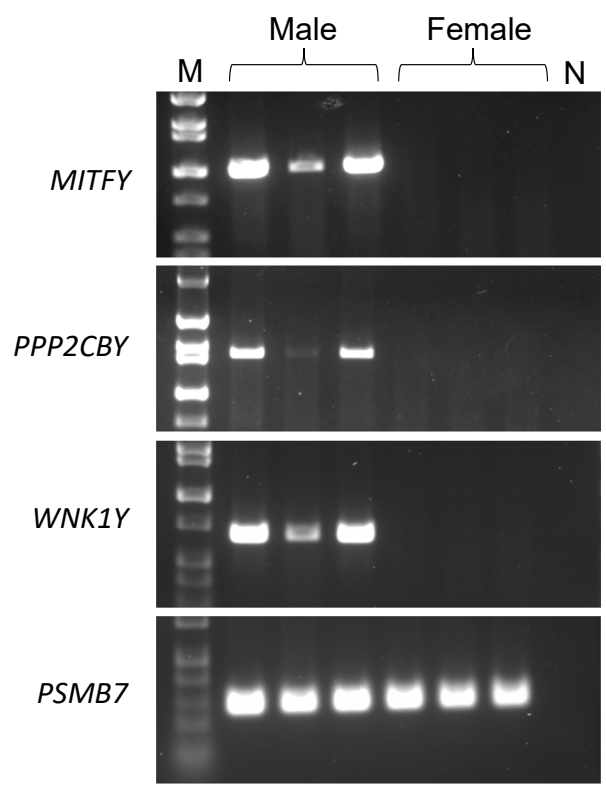

Figure 3. Primers were designed to detect Y-linked retrocopies of three genes, as well as the presence of a control autosomal gene (PSMB7). Polymerase chain reaction using DNA from 3 male dogs and 3 female dogs showed male specific amplification for the 3 retrocopies (top three panels). Amplification for all dogs is shown for PSMB7 in the bottom panel.

\subsection{MITFY}

We Sanger sequenced $944 \mathrm{bp}$ of MITFY from genomic DNA and used overlapping next-generation sequencing reads to determine the $5^{\prime}$ and $3^{\prime}$ sequences. Parental MITF, located on CFA20, and MITFY are $98 \%$ identical (Figure S1). The retrocopy is consistent with human MITF isoform A1, which uses exon $1 \mathrm{~A}$ and encodes a 526 aa protein. However, MITFY harbors a single base insertion that predicts a frameshift and premature stop codon that would truncate the protein by nearly $40 \%$ (Figure 4 ).

The MITFY primer set amplified transcripts arising from both the parental gene and retrogene. cDNA amplicons were obtained from all four tested tissues, sequenced, and genotyped for Y-specific variants. In testis, we detected expression of MITF and MITFY, evidenced by heterozygosity for $\mathrm{Y}$ chromosome and autosomal alleles. Heart, liver, and brain only showed expression of the autosomal copy.

MITF retrogene sequences were also identified in WGS from males of older Canidae family members: Coyote (Canis latrans), African wild dog (Lycaon pictus), and island fox (Urocyon littoralis) (Figure 5). Red foxes (Vulpes vulpes) did not show evidence of a retrogene based on WGS. The domestic dog, coyote, and African wild dog shared a majority of MITFY variants, while the island fox possessed a unique variant haplotype. 


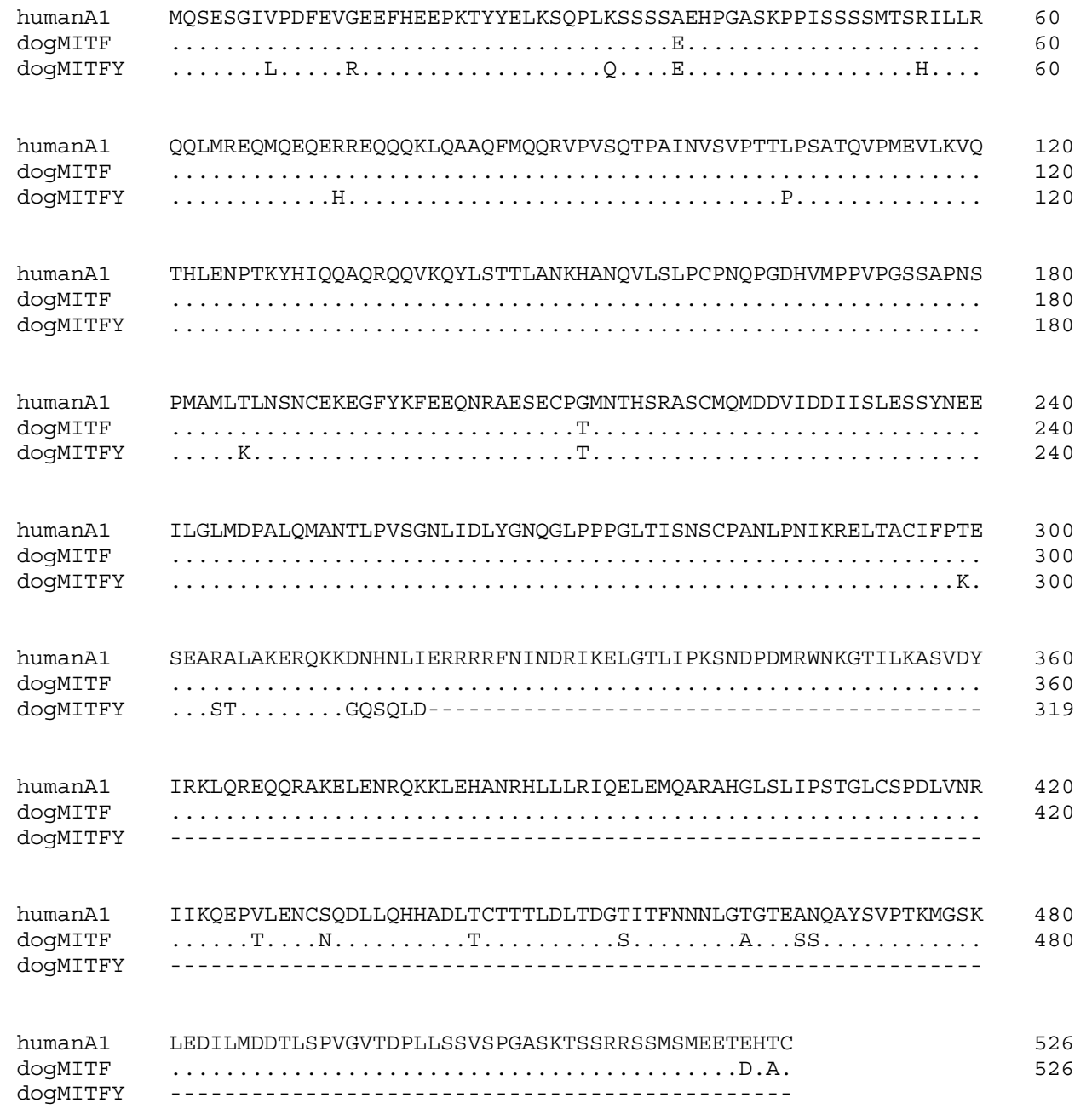

Figure 4. Alignment of MITF protein sequences: Human isoform A1, canine autosomal (CFA20), and predicted $Y$ retrogene sequences.

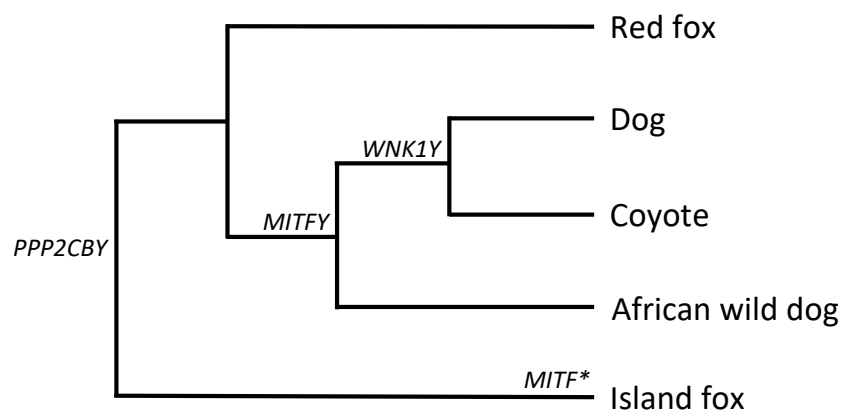

Figure 5. Cladogram (based on [12]) depicting the most parsimonious hypothesis for the retrotransposition of three genes in the Canidae family. *The MITF retrocopy in the island fox could not definitively be placed on the Y chromosome.

\section{2. $P P P 2 C B Y$}

We Sanger sequenced $\sim 1330$ bp of PPP2CBY from genomic DNA and determined the $5^{\prime}$ and $3^{\prime}$ sequences using WGS data. $P P P 2 C B Y$ is $89 \%$ identical to parental $P P P 2 C B$, located on CFA16 (Figure S2). The reverse $P$ PP2CBY primer contains a $9 \mathrm{bp}$ Y-specific deletion, and thus PCR was specific to the $Y$ chromosome copy in both gDNA and cDNA. We found no evidence of expression of 
$P P P 2 C B Y$ in any of the four tissues examined and posit that it is a pseudoretrogene. Genomic evidence of $P P P 2 C B Y$ was present in all male (but not female) canid genomes investigated herein (Figure 5).

\subsection{WNK1Y}

We Sanger sequenced $668 \mathrm{bp}$ of WNK1Y from genomic DNA and used overlapping next-generation sequencing reads to determine the $5^{\prime}$ and $3^{\prime}$ sequences of the retrocopy. The WNK1Y retrocopy has no identifiable translational start site and is composed of exons 25,26 , and a spliced version of exon 27. WNK1Y primers amplified transcripts arising from both the parental gene and retrogene. cDNA amplicons were obtained from testis and heart tissues. Sequencing of amplicons showed that WNK1Y transcripts were present in both tissues.

Using BLAT, we discovered that all $1041 \mathrm{bp}$ of WNK1Y align with $95.4 \%$ similarity to a continuous fragment of an unplaced scaffold: chrUn_AAEX03024224 (chrUn hereafter). Our original WNK1Y primer set, used to show male-specific amplification, did not amplify the chrUn sequences because of diverged nucleotides in the primer sequences. We designed new primers sets within and flanking the WNK1 sequences on chrUn that amplified in both males and females (data not shown), thereby confirming that the chrUn retrocopy is autosomal. Sequence alignment of the chr27 parent WNK1, WNK1Y, and chrUn WNK1 revealed five shared SNPs between the two retrocopies (Figure S3). Using IGV, we observed signatures of a Y chromosome haplotype beyond WNK1, across approximately $6500 \mathrm{bp}$ of the $9600 \mathrm{bp}$ chrUn scaffold, in both dog and coyote.

We also observed many chrUn WNK1 reads having mate pairs mapping to CFA34, corresponding to ETV5. We visually inspected WGS flanking WNK1 and identified an ETV5 retrocopy containing exons 3, 4, and 5 and partial intron 5. WNK1 and ETV5 are arranged in a head-to-head orientation. Using an ETV5 forward primer designed using changes on the $Y$ haplotype and a WNK1Y reverse, we observed male-specific amplification (data not shown), indicating that like WNK1, ETV5 retrocopies exist on both chrUn and the $\mathrm{Y}$ chromosome. The primers did not yield amplicons from cDNA.

\section{Discussion}

A GWAS revealed 50 SNPs significantly associated with sex, 49 of which are in perfect linkage disequilibrium, despite mapping to 10 different chromosomes. Based on the data presented here, we posit that these SNPs represent autosome or X chromosome sequences that are duplicated on the $\mathrm{Y}$ chromosome. Both sexes possess two wild-type copies, while males also have a diverged Y-chromosome copy for which they are hemizygous. When aligned to the female reference genome, the Y-chromosome sequences align with the homologous sequences, making the diverged nucleotides appear to be SNPs that are highly polymorphic across breeds and other canids. Thus, 47 of the 49 Y-linked SNPs revealed in our GWAS were misplaced on the genome map. The two SNPs correctly placed on the $\mathrm{Y}$ chromosome likely have a homologous autosomal or $\mathrm{X}$ chromosome sequence, causing monomorphic genotypes in SNP array data from females.

In this study, we focused on the Y-linked SNPs that map to an autosomal gene and discovered novel canid retrocopies, two of which show transcriptional activity. In dogs, two other expressed retrogenes have been associated with common skeletal malformations; both originate from fibroblast growth factor 4 (FGF4) on CFA18 [20-22]. Additional studies based on the Ensembl annotation of the canine reference genome suggest that there are between 95 and 409 retrogenes in dogs, but none are confirmed to be expressed other than the FGF4 retrogenes [23-25].

Here, we confirmed transcription of MITFY in testis, although it is not clear whether the retrogene produces a functional protein. We found no evidence for novel introns within MITFY, thus the premature stop codon is unlikely to trigger nonsense mediated decay if it is translated [26,27]. The parental gene, MITF, encodes a transcription factor involved in the development of various cell types, most notably melanocytes [28]. The predicted premature stop codon in the Y retrogene occurs within the bHLH-LZ domain, which would normally permit DNA binding of target genes [28]. However, exons 1A and 4, which enable transactivational activity [29], precede the MITFY translational termination signal. 
At the mRNA level, MITF is a known target of several miRNAs [30]. Though we observed numerous retrogene-specific variants in the $3^{\prime}$ UTR, we found that many miRNA bindings sites are preserved between parental MITF and the retrogene. Without additional studies, the activity of MITFY and its impact on the expression and/or function of parental MITF is unclear.

WNK1Y transcripts were present in testis and cardiac tissue. WNK1 is a serine-threonine kinase encoded by a 33 exon gene with multiple tissue-specific isoforms [31]. WNK1Y is composed of exons 25,26 , and parts of exon 27 , a splicing pattern inconsistent with previously described isoforms and for which we cannot find a clear translational start site. Immediately upstream of WNKY is an ETV5Y retrocopy in reverse orientation. The parent gene, ETV5, is an ETS transcription factor important in morphogenesis and fertility [32]. ETS transcription factors have a role in oncogenesis and ETV5 gene fusions have been described in prostate cancers [33]. ETV5Y consists of three exons, part of an intron, and lacks the translational start site. We also found WNK1 and ETV5 retrocopies in the same head-to-head orientation on an unplaced scaffold, chrUn_AAEX03024224. Examination of the remainder of the scaffold revealed a male-specific haplotype in the dog and coyote, suggesting that the unplaced scaffold is duplicated, at least in part, on their Y chromosomes.

Our data are inconclusive with regard to the chromosomal location of the island fox MITF retrocopy, which did not share variants with MITFY. Unfortunately, WGS from a female of the same subspecies was not available to eliminate or confirm the presence of an autosomal retrocopy event. Regardless, we hypothesize that the island fox retrocopy is an independent event and that MITFY arose after the divergence of the red fox. Independent retrocopy events stemming from the same parental gene have been reported numerous times [4,25,34]. Each of the parental genes of the retrocopies identified herein has at least one other retrocopy insertion event in another species [35,36]. PPP2CB retrogenes have been identified in several mammals, with some species having multiple copies and showing transcriptional activity [36]. That distinct lineages have had retrocopies arising from the same genes and independently driven to fixation suggests that these events may have conferred increased fitness.

Like FGF4, the parent genes of the novel retrocopies identified herein all have roles in spermatogenesis [29,37-39]. Studies in other organisms have similarly observed that parental genes often have male-specific functions [34,40]. It has been suggested that transcripts present during spermatogenesis may be more likely to become retrocopies because of the open configuration of chromatin during periods of high transcriptional activation [41]. This may also underlie the observation that retrogenes are often expressed in testes [41], as we observed with MITFY and WNK1Y.

We did not find evidence for novel X chromosome derived retrogenes. Two of the Y-linked SNPs mapped to the $\mathrm{X}$ chromosome are in a previously described $120 \mathrm{~kb} \mathrm{X}$ to $\mathrm{Y}$ chromosome transposition event in dogs that includes TRAPPC2 and OFD1 [15]. Manual inspection in IGV confirmed the presence of this duplication in all canid sequences investigated herein. A duplication of OFD1 and portions of TRAPPC 2 and GPM6B has been described in pigs, with high OFD1Y expression observed in porcine testis [42], and OFD1 transposition to $Y$ has also been described in primates [43]. The frequent transposition of OFD1 to the $\mathrm{Y}$ chromosome in mammals may indicate an acquired function in spermatogenesis [42]. A sex-linked Illumina SNP was also observed in SHROOM2, located in the pseudoautosomal region of the $X$ chromosome [15]. We detected both exonic and intronic variants on a shared male-specific haplotype, suggesting that like OFD1, the entire gene has been duplicated onto the canid Y.

\section{Conclusions}

We have shown that 47 SNPs from the Illumina CanineHD 230K are actually variations in homologous sequences located on the $\mathrm{Y}$ chromosome. This work illustrates that retrocopies and other genetic duplications are a source of false variant calls, a complication of using short read technologies and/or having an incomplete reference genome. We further investigated 12 SNPs mapping to expressed autosomal sequences and identified four novel canid $\mathrm{Y}$ chromosome retrocopies, two 
having transcriptional activity. The remaining SNPs mark chromosomal segments that have likely been duplicated on the Y chromosome, including nearly $300 \mathrm{~kb}$ of chromosome 19 that harbors two lincRNAs. Additional research into these regions holds promise for the identification of unique $Y$ chromosome sequences. Complete sequence and annotation of the canine $Y$ chromosome would be a valuable resource for researchers of multifactorial traits, particularly those that show a sex predilection such as heart, autoimmune, and infectious diseases.

Supplementary Materials: The following are available online at http://www.mdpi.com/2073-4425/10/4/320/s1, Figure S1: title, Table S1: title, Video S1: title. The following are available online: Figure S1: Alignment of MITFY and parental MITF (Chr20) sequences, the start and stop codons are bolded, Illumina SNPs are highlighted in yellow; Figure S2: Alignment of PPP2BCY and parental PPP2CB (Chr16) sequences, the start and stop codons are bolded, Illumina SNPs are highlighted in yellow; Figure S3: Alignment of WNK1 sequences: parent gene (Chr27), Y chromosome retrogene, and chrUn_AAEX03024224 retrocopy, Illumina SNPs are highlighted in yellow; Table S1: Sequence Read Archive accession numbers for resequencing data used herein.

Author Contributions: Conceptualization, J.E., K.T. and L.C.; data curation, K.T., L.C. and R.N.; investigation, A.S., J.E., K.T., L.C., writing—original draft, J.E., K.T., L.C. and R.N., writing—review and editing, A.S., J.E., K.T. and L.C.

Funding: This research received no external funding.

Acknowledgments: The authors wish to thank Dr. Mike Vaughan for critical reading of the manuscript.

Conflicts of Interest: The authors declare no conflict of interest.

\section{References}

1. Skaletsky, H.; Kuroda-Kawaguchi, T.; Minx, P.J.; Cordum, H.S.; Hillier, L.; Brown, L.G.; Repping, S.; Pyntikova, T.; Ali, J.; Bieri, T.; et al. The male-specific region of the human Y chromosome is a mosaic of discrete sequence classes. Nature 2003, 423, 825-837. [CrossRef]

2. Yang, Y.; Chang, T.C.; Yasue, H.; Bharti, A.K.; Retzel, E.F.; Liu, W.S. ZNF280BY and ZNF280AY: autosome derived Y-chromosome gene families in Bovidae. BMC Genomics 2011, 13. [CrossRef]

3. Dyer, K.A.; White, B.E.; Bray, M.J.; Piqué, D.G.; Betancourt, A.J. Molecular evolution of a Y chromosome to autosome gene duplication in Drosophila. Mol. Biol. Evol. 2011, 28, 1293-1306. [CrossRef]

4. Hughes, J.F.; Skaletsky, H.; Koutseva, N.; Pyntikova, T.; Page, D.C. Sex chromosome to autosome transposition events counter Y-chromosome gene loss in mammals. Genome Biol. 2015, 16. [CrossRef] [PubMed]

5. Page, D.C.; Harper, M.E.; Love, J.; Botstein, D. Occurence of a transposition from the X-chromosome long arm to the Y-chromosome short arm during human evolution. Nature 1984, 311, 119-123. [CrossRef] [PubMed]

6. Saxena, R.; Brown, L.G.; Hawkins, T.; Alagappan, R.K.; Skaletsky, H.; Reeve, M.P.; Reijo, R.; Rozen, S.; Dinulos, M.B.; Disteche, C.M.; Page, D.C. The DAZ gene cluster on the human Y chromosome arose from an autosomal gene that was transposed, repeatedly amplified and pruned. Nat. Genet. 1996, 14, $292-299$. [CrossRef] [PubMed]

7. Lahn, B.T.; Page, D.D. Retroposition of autosomal mRNA yielded testis-specific gene family on human $Y$ chromosome. Nat. Genet. 1999, 21, 429-433. [CrossRef]

8. Cao, P.; Wang, L.; Jiang, Y.; Yi, Y.; Qu, F.; Liu, T.; Lv, L. De Novo origin of VCY2 from autosome to y transposed amplicon. PLoS ONE 2015, 10, e0119651. [CrossRef]

9. Carvalho, A.B.; Vicoso, B.; Russo, C.A.M.; Swenor, B.; Clark, A.G. Birth of a new gene on the Y chromosome of Drosophila melanogaster. Proc. Natl. Acad. Sci. USA 2015, 112, 12450-12455. [CrossRef]

10. Marques, A.C.; Dupanloup, I.; Vinckenbosch, N.; Reymond, A.; Kaessmann, H. Emergence of young human genes after a burst of retroposition in primates. PLoS ONE 2005, 3, e357. [CrossRef]

11. Bai, Y.; Casola, C.; Betrán, E. Evolutionary origin of regulatory regions of retrogenes in Drosophila. BMC Genomics 2008, 9, 241. [CrossRef] [PubMed]

12. Lindblad-Toh, K.; Wade, C.M.; Mikkelsen, T.S.; Karlsson, E.K.; Jaffe, D.B.; Kamal, M.; Clamp, M.; Chang, J.L.; Kulbokas, E.J., III; Zody, M.C.; et al. Genome sequence, comparative analysis and haplotype structure of domestic dog. Nature 2005, 438, 803-819. [CrossRef] [PubMed]

13. Hoeppner, M.P.; Lundquist, A.; Pirun, M.; Meadows, J.R.S.; Zamani, N.; Johnson, J.; Sundström, G.; Cook, A.; FitzGerald, M.G.; Swofford, R.; Mauceli, E.; et al. An improved canine genome and a comprehensive catalogue of coding genes and non-coding transcripts. PLoS ONE 2014, 9, e91172. [CrossRef] [PubMed] 
14. Natanaelsson, C.; Oskarsson, M.C.R.; Angleby, H.; Lundeberg, J.; Kirkness, E.; Savolainen, P. Dog Y chromosomal DNA sequence: identification, sequencing and SNP discovery. BMC Genet. 2006, 7, 45. [CrossRef] [PubMed]

15. Li, G.; Davis, B.W.; Raudsepp, T.; Pearks Wilkerson, A.J.; Mason, V.C.; Ferguson-Smith, M.; O’Brien, P.C.; Waters, P.D.; Murphy, W.J. Comparative analysis of mammalian Y chromosomes illuminates ancestral structure and lineage-specific evolution. Genome Res. 2013, 23, 1486-1495. [CrossRef] [PubMed]

16. Bolger, A.M.; Lohse, M.; Usadel, B. Trimmomatic: a flexible trimmer for Illumina sequence data. Bioinformatics 2014, 30, 2114-2120. [CrossRef] [PubMed]

17. Wu, T.D.; Nacu, S. Fast and SNP-tolerant detection of complex variants and splicing in short reads. Bioinformatics 2010, 7, 873-881. [CrossRef]

18. Li, H. A statistical framework for SNP calling, mutation discovery, association mapping and population genetical parameter estimation from sequencing data. Bioinformatics 2011, 27, 2987-2993. [CrossRef]

19. Thorvaldsdóttir, H.; Robinson, J.T.; Mesirov, J.P. Integrative genomics viewer (IGV): high-performance genomics data visualization and exploration. Brief bioinform. 2013, 14, 178-192. [CrossRef]

20. Parker, H.G.; VonHoldt, B.M.; Quignon, P.; Margulies, E.H.; Shao, S.; Mosher, D.S.; Spady, T.C.; Elkahloun, A.; Cargill, M.; Jones, P.G.; et al. An expressed fgf4 retrogene is associated with breed-defining chondrodysplasia in domestic dogs. Science 2009, 325, 995-998. [CrossRef]

21. Brown, E.A.; Dickinson, P.J.; Mansour, T.; Sturges, B.K.; Aguilar, M.; Young, A.E.; Korff, C.; Lind, J.; Ettinger, C.L.; Varon, S.; et al. FGF4 retrogene on CFA12 is responsible for chondrodystrophy and intervertebral disc disease in dogs. Proc. Natl. Acad. Sci. USA 2017, 114, 11476-11481. [CrossRef] [PubMed]

22. Marchant, T.W.; Johnson, E.J.; McTeir, L.; Johnson, C.I.; Gow, A.; Liuti, T.; Kuehn, D.; Svenson, K.; Bermingham, M.L.; Drögemüller, M.; et al. Canine Brachycephaly is associated with a retrotransposon-mediated missplicing of SMOC2. Curr. Biol. 2017, 27, 1573-1584. [CrossRef] [PubMed]

23. Yu, Z.; Morais, D.; Ivanga, M.; Harrison, P.M. Analysis of the role of retrotransposition in gene evolution in vertebrates. BMC Bioinformatics 2007, 8, 308. [CrossRef] [PubMed]

24. Potrzebowski, L.; Vinckenbosch, N.; Marques, A.C.; Chalmel, F.; Jégou, B.; Kaessmann, H. Chromosomal gene movement reflect the recent origin and biology of therian sex chromosomes. PLoS Biol. 2008, 6, e80. [CrossRef]

25. Pan, D.; Zhang, L. Burst of young retrogenes and independent retrogene formation in mammals. PLoS ONE 2009, 4, e5040. [CrossRef] [PubMed]

26. Fablet, M.; Bueno, M.; Potrzebowski, L.; Kaessmann, H. Evolutionary origin and function of retrogene introns. Mol. Biol. Evol. 2009, 26, 2147-2156. [CrossRef] [PubMed]

27. Lindeboom, R.G.; Supek, F.; Lehner, B. The rules and impact of nonsense mediated RNA decay in human cancers. Nat. Genet. 2016, 48, 1112-1118. [CrossRef]

28. Hemesath, T.J.; Steingrímsson, E.; McGill, G.; Hansen, M.J.; Vaught, J.; Hodgkinson, C.A.; Arnheiter, H.; Copeland, N.G.; Jenkins, N.A.; Fisher, D.E. Microphthalmia, a critical factor in melanocyte development, defines a discrete transcription factor family. Genes. Dev. 1994, 8, 2770-2780. [CrossRef]

29. Saito, H.; Takeda, K.; Yasumoto, K.; Ohtani, H.; Watanabe, K.; Takahashi, K.; Fukuzaki, A.; Arai, Y.; Yamamoto, H.; Shibahara, S. Germ cell-specific expression of microphthalmia associated transcription factor mRNA in mouse testis. J. Biochem. 2003, 134, 143-150. [CrossRef]

30. Hartman, M.L.; Czyz, M. MITF in melanoma: mechanisms behind its expression and activity. Cell. Mol. Life Sci. 2015, 72, 1249-1260. [CrossRef]

31. Vidal-Petiot, E.; Cheval, L.; Faugeroux, J.; Malard, T.; Doucet, A.; Jeunemaitre, X.; Hadchouel, J. A new methodology for quantification of alternatively spliced exons reveals a highly tissue-specific expression pattern of WNK1 isoforms. PLoS ONE 2012, 7, e37751. [CrossRef]

32. Eo, J.; Song, H.; Lim, H.J. Etv5, a transcription factor with versatile functions in male reproduction. Clin. Exp. Reprod. Med. 2012, 39, 41-45. [CrossRef] [PubMed]

33. Oh, S.; Shin, S.; Janknecht, R. ETV1, 4 and 5: an oncogenic subfamily of ETS transcription factors. Biochim. Biophys. Acta 2012, 1826, 1-12. [CrossRef]

34. Bai, Y.; Casola, C.; Feschotte, C.; Betrán, E. Comparative genomics reveals a constant rate of origination and convergent acquisition of functional retrogenes in Drosophila. Genome Biol. 2007, 8, R11. [CrossRef]

35. Mouse Genome Sequencing Consortium. Initial sequencing and comparative analysis of the mouse genome. Nature 2002, 420, 520-562. [CrossRef] 
36. Rosikiewicz, W.; Kabza, M.; Kosiński, J.G.; Ciomborowska-Basheer, J.; Kubiak, M.R.; Makałowska, I. Retrogene DB - a database of plant and animal retrocopies. Database 2017, 2017, 1-11. [CrossRef]

37. O’Bryan, M.K.; Grealy, A.; Stahl, P.J.; Schlegel, P.N.; McLachlan, R.I.; Jamsai, D. Genetic variants in the ETV5 gene in fertile and infertile men with nonobstructive azoospermia associated with Sertoli cell-only syndrome. Fertil. Steril. 2012, 98, 827-835. [CrossRef]

38. Su, Y.; Sugiura, K.; Sun, F.; Pendola, J.K.; Cox, G.A.; Handel, M.A.; Schimenti, J.C.; Eppig, J.J. MARF1 regulates essential oogenic processes in mice. Science 2012, 335, 1496-1499. [CrossRef]

39. Shekarabi, M.; Lafrenière, R.G.; Gaudet, R.; Laganière, J.; Marcinkiewicz, M.M.; Dion, P.A.; Rouleau, G.A. Comparative analysis of the expression profile of Wnk1 and Wnk1/Hsn2 splice variants in developing and adult mouse tissues. PLoS ONE 2013, 8, e57807. [CrossRef] [PubMed]

40. Rohozinski, J. Lineage-independent retrotransposition of UTP14 associated with male fertility has occurred multiple times throughout mammalian evolution. R. Soc. Open Sci. 2017, 4, 171049. [CrossRef] [PubMed]

41. Ciomborowska, J.; Rosikiewicz, W.; Szklarczyk, D.; Makałowski, W.; Makałowska, I. “Orphan” retrogenes in the human genome. Mol. Biol. Evol. 2012, 30, 384-396. [CrossRef] [PubMed]

42. Skinner, B.M.; Sargent, C.A.; Churcher, C.; Hunt, T.; Herrero, J.; Loveland, J.E.; Dunn, M.; Louzada, S.; Fu, B.; Chow, W. The pig X and Y chromosomes: structure, sequence, and evolution. Genome Res. 2016, 26, 130-139. [CrossRef] [PubMed]

43. Chang, T.C.; Klabnik, J.L.; Liu, W.S. Regional selection acting on the OFD1 gene family. PLoS ONE 2011, 6, e26195. [CrossRef] [PubMed]

(C) 2019 by the authors. Licensee MDPI, Basel, Switzerland. This article is an open access article distributed under the terms and conditions of the Creative Commons Attribution (CC BY) license (http://creativecommons.org/licenses/by/4.0/). 